\title{
Lidil
}

Revue de linguistique et de didactique des langues

$52 \mid 2015$

Les pratiques artistiques dans l'apprentissage des langues

\section{L'enseignement par le théâtre en classe de français au Québec : état des lieux et pistes didactiques}

Didactic Approaches to Language Education through Theatre in French Class in Quebec

Joannie Dubois et Ophélie Tremblay

\section{OpenEdition}

Journals

Édition électronique

URL : https://journals.openedition.org/lidil/3863

DOI : 10.4000/lidil.3863

ISSN : 1960-6052

Éditeur

UGA Éditions/Université Grenoble Alpes

Édition imprimée

Date de publication : 20 novembre 2015

Pagination : 129-152

ISBN : 978-2-84310-312-4

ISSN : $1146-6480$

Référence électronique

Joannie Dubois et Ophélie Tremblay, «L'enseignement par le théâtre en classe de français au Québec état des lieux et pistes didactiques », Lidil [En ligne], 52 | 2015, mis en ligne le 01 janvier 2017, consulté le 28 juin 2022. URL : http://journals.openedition.org/lidil/3863 ; DOI : https://doi.org/10.4000/lidil. 3863 


\title{
L'enseignement par le théâtre en classe de français au Québec : état des lieux et pistes didactiques
}

\author{
Joannie Dubois* et Ophélie Tremblay**
}

\begin{abstract}
Résumé
Le genre théâtral est souvent considéré comme un «objet de réticence» en classe de français (Dardaillon, 2009). Malgré l'évidence de son caractère dynamique (Audet \& Mercier, 2004) et la qualité des œuvres produites, notamment au Québec, le théâtre est délaissé au profit de différents genres, dont le roman, largement exploité dans les classes (Dardaillon, 2009). Peu de recherches se sont penchées sur les raisons qui pourraient expliquer ce désintérêt, et peu d'études encore proposent un état des lieux en matière d'enseignement du français par le théâtre en contexte québécois. Néanmoins, un certain nombre d'hypothèses peuvent être formulées pour expliquer la sous-exploitation de l'activité théâtrale en classe de français au Québec. Cette contribution a pour objectif de présenter ces hypothèses et de proposer quelques solutions didactiques adaptées à l'enseignement secondaire (élèves âgés entre 11 et 17 ans). L'article se développe en trois parties : (1) la pertinence d'intégrer le théâtre en classe de langue; (2) une analyse du contenu relatif au théâtre dans les programmes de français du MELS (Ministère de l'Éducation, du Loisir et du Sport); (3) des pistes didactiques permettant de répondre aux difficultés d'exploitation du genre théâtral en classe : le sous-texte, le Théâtre des lecteurs, le texte blanc et la dramatisation.
\end{abstract}

* Étudiante à la maitrise en didactique des langues, Université du Québec à Montréal (Québec) \& Centre de recherche interuniversitaire sur la formation et la profession enseignante (CRIFPE).

** Professeure, Département de didactique des langues, Université du Québec à Montréal (Québec) \& Centre de recherche interuniversitaire sur la formation et la profession enseignante (CRIFPE). 


\begin{abstract}
Theatre, as a literary genre, is considered unpopular in the French classrooms (Dardaillon, 2009). Despite its obvious dynamic nature (Audet \& Mercier, 2004) and the quality of the texts written, notably in the province of Quebec, theatre as a literary genre is neglected in comparison to other such as the novel, which is widely exploited in class (Dardaillon, 2009). According to our actual knowledge of the situation, not many researches show the reasons behind this possible lack of interest towards the theatre genre and the use or theatrical activities in the classroom. However, it is conceivable to formulate some hypotheses in order to explain the possible underuse of theatrical activities in the French classroom. The following article will present these hypotheses and propose some didactic solutions, adapted to classroom realities. In order to present those components, the upcoming text will be divided in three parts: (1) the relevance to include theatre in the language classroom; (2) an analysis of the reference programs (MELS - Ministère de l'Éducation et du Sport) used by French teachers for the teaching of theatre; (3) didactic solutions as an answer to possible conducting difficulties (Readers' theatre, dramatization, comprehensive reading and interpretation of the blank text).
\end{abstract}

\title{
1. Introduction
}

La contribution que nous présentons est issue de réflexions que nous menons depuis plus d'un an sur la question du théâtre et de son enseignement au Québec, ainsi que sur celle de l'utilisation du théâtre comme moyen d'enseignement en classe de français langue première au secondaire. La première auteure de cet article détient une formation en enseignement de l'art dramatique et enseigne de façon ponctuelle dans plusieurs classes du secondaire, tout en poursuivant des études de deuxième cycle en didactique des langues (sous la direction de la deuxième auteure de cet article, dont un certain nombre de recherches portent sur la didactique de l'oral). À travers ses expériences d'enseignement, elle a pu observer que le théâtre était bien souvent malaimé des enseignants en classe de français au secondaire. Pourtant, dès qu'elle proposait une activité théâtrale pour travailler, par exemple, une des facettes de la compétence à communiquer oralement (débit, volume, articulation, etc.), elle remarquait l'enthousiasme et l'intérêt des élèves à plonger dans l'expérience théâtrale. Ces constats amènent à se questionner sur l'exploitation sans doute insuffisante du théâtre comme objet et comme moyen d'enseignement en classe de français. 
Le présupposé sur lequel prend appui cette contribution est que le théâtre est peu ou mal connu par les enseignants du français du secondaire au Québec, pour au moins trois raisons. D'abord, nous pensons que les enseignants de français au secondaire n'ont peut-être pas reçu la formation didactique nécessaire à une intégration de l'activité théâtrale dans leur enseignement du français, ce que nous tenterons de montrer plus loin, à travers une analyse des programmes de formation universitaire en enseignement du français au secondaire. Nous montrerons également que, si les programmes ministériels pour la formation des élèves du niveau secondaire intègrent bien la question du théâtre, ils proposent peu de pistes didactiques concrètes pour travailler le théâtre en classe. Enfin, nous pensons que certaines contraintes techniques (espace restreint, nombre d'élèves élevé, etc.) freinent son utilisation comme moyen d'enseignement en classe.

Ces pistes de réflexion structurent notre contribution, qui se développe en trois parties. Nous rappelons d'abord la pertinence d'enseigner le français par le théâtre, en nous appuyant pour ce faire sur des résultats de recherches menées en contexte d'enseignement de l'anglais langue première. Nous dressons ensuite un état des lieux de l'enseignement du théâtre au niveau secondaire québécois, à partir d'une analyse des programmes ministériels et des contenus des programmes de formation des enseignants de français au secondaire. La troisième partie de l'article est consacrée à diverses propositions didactiques qui s'appuient sur les résultats de recherche présentés en première partie et qui répondent aux indications des programmes en matière d'enseignement du français par le théâtre. Ces pistes visent également à contourner certaines contraintes techniques (que nous évoquerons brièvement) et à redonner ses lettres de noblesse à l'activité théâtrale en classe de français au Québec, à travers une approche ludique visant à la fois le plaisir de jouer et le plaisir d'apprendre la langue. Bien que ces réflexions et ces pistes didactiques s'inscrivent plus particulièrement dans le contexte de l'enseignement du français langue maternelle au Québec, nous estimons qu'elles sont tout aussi pertinentes pour l'enseignement du français langue maternelle en dehors du Québec (France, Suisse, Belgique, etc.) et pour l'enseignement du français langue étrangère ou seconde. 


\section{Pertinence d'enseigner par le théâtre en classe de français}

Une des hypothèses qui sous-tend la réflexion proposée dans cette contribution est qu'une activité d'ordre artistique jumelée à une activité de langue permettra à l'élève de développer une multitude de compétences (Meirieu, 2002; Sousa, 2004; St-Cœur, 2004), par exemple exercer son jugement critique (Aden, 2009), mettre en œuvre sa pensée créatrice $(\mathrm{ibid}$.), communiquer de façon appropriée (Fonio \& Genicot, 2011; Magalhaes dos Reis, 2008), etc. Plus particulièrement, les activités qui font appel au théâtre, en tant que moyen d'enseignement, sont susceptibles de contribuer au développement de ces compétences. Nous distinguons ainsi l'enseignement du thêâtre lui-même (étude du rapport texte/spectacle, théorie du texte, etc.) de l'enseignement par le théâtre (recours à des activités d'ordre artistique en classe afin de faire progresser les connaissances et les compétences des élèves en lien avec la matière, ici le français). C'est davantage la perspective d'un enseignement du français par le théâtre qui sera adoptée dans le cadre du présent article.

L'enseignement par le théâtre présente en effet plusieurs avantages. Par exemple, dans une perspective plus large que celle de la classe de français, l'intégration d'activités d'ordre artistique à l'école (telles que le théâtre) est très bénéfique pour l'apprenant. De telles activités auraient un impact positif sur les apprentissages réalisés dans les matières scolaires, notamment en sciences (Dogru, 2010). Elle joueraient également un rôle positif dans le développement des compétences sociales de l'élève, plus précisément en ce qui a trait à l'empathie, à la régulation des émotions (Winner, Goldstein \& Vincent-Lancrin, 2014) et à l'estime de soi (Kamaruddin, 2010). D'autres recherches ont également montré que la pratique d'une activité artistique à l'école aurait un effet sur l'épanouissement complet de la personne (Meirieu, 2002; Sousa, 2004 ; St-Cœur, 2004) et qu'elle affecterait positivement la motivation de l'apprenant (Sousa, 2004; St-Cœur, 2004), en plus d'être une source de plaisir (Kararuddin \& Kamarul Kabilan, 2010).

L'enseignement par le théâtre contribue également au développement des compétences linguistiques. Une activité aussi simple que le Théâtre des lecteurs, qui consiste en une lecture à voix haute et en groupe d'un texte ou d'un extrait de texte de théâtre, à la suite de laquelle il y aura ou non une présentation devant public, aurait un impact sur la fluidité en lecture des élèves (Kararuddin \& Kamarul Kabilan, 2010; MacFadden, 2010). 
Une étude (Smith, 2011) a mis en évidence que l'activité du Théâtre des lecteurs, sans prétendre être le moyen le plus efficace de faire progresser les élèves en fluidité de lecture, en facilitait grandement le développement. Dans cette recherche quasi expérimentale, deux groupes ont été exposés à une activité du Théâtre des lecteurs sur une période de dix semaines. Pendant la même période, deux groupes contrôle ont participé à une activité de lecture répétée. Au terme de l'expérimentation, les élèves des groupes expérimentaux et contrôles ont amélioré significativement leur performance en termes de fluidité en lecture. Les élèves des groupes expérimentaux ont plus spécifiquement fait preuve d'une amélioration à l'égard de leur attitude face à la lecture de nature récréative, c'est-à-dire les lectures non obligatoires.

Une recherche réalisée par MacFadden (2006) a également porté sur la pratique du Théâtre des lecteurs. Durant 9 semaines, à raison d'une fois par semaine, trois artistes invités sont venus animer, à tour de rôle, une activité dans une classe expérimentale pendant une période d'environ une heure. Pendant cette même période, le groupe contrôle n'a pas eu accès à ces activités. Cette expérimentation a conduit aux résultats suivants : (1) les élèves ayant participé aux activités d'ordre artistique avaient de meilleures capacités de rétroaction, c'est-à-dire la formulation de commentaires faisant suite à une activité ; (2) tous les élèves, sauf un, ont affirmé avoir aimé les activités; (3) les enseignants ont relevé, par observation, que leurs élèves semblaient avoir développé leurs habiletés à utiliser un vocabulaire plus riche et varié, qu'ils avaient amélioré leurs capacités de rétroaction, qu'ils étaient intéressés et engagés lors des activités. La chercheure ne s'avance pas sur les raisons de cette amélioration du vocabulaire, mais nous pouvons émettre l'hypothèse que les élèves ont eu l'occasion de s'exprimer dans un contexte authentique qui stimulait leur utilisation du vocabulaire. Par ailleurs, les enseignants ont aussi relevé que des élèves plus âgés (par exemple, des adolescents) auraient été davantage en mesure d'apprécier les activités artistiques en raison d'une plus grande maturité.

Enfin, selon La Von Bridges (2006), la pratique du Théâtre des lecteurs aurait une influence sur le développement de la compétence orale, en plus d'entrainer des répercussions positives sur la motivation, le plaisir à la tâche et le sentiment d'efficacité personnel des élèves.

Les résultats de ces études sont éclairants quant à l'impact positif que peuvent avoir des activités de nature théâtrale, non seulement sur l'apprentissage de la langue et le développement des compétences linguistiques, mais aussi sur des facteurs clés de la réussite scolaire, notamment 
la motivation, l'estime de soi et le développement d'attitudes positives face à l'apprentissage (Sousa, 2002; St-Cœur, 2004; Aden, 2009). Enfin, l'activité artistique renforcerait le rapport à la culture de l'élève sous les dimensions épistémiques, subjectives (Simard, 2004) et sociales (Charlot, 1997), ce qui est susceptible de se répercuter favorablement sur le développement des compétences en français.

Les recherches que nous avons rapportées jusqu'à présent ont été menées principalement en anglais aux États-Unis auprès d'élèves anglophones. Dans notre revue des écrits sur la question du théâtre et de son exploitation didactique, nous avons trouvé très peu d'études menées en contexte québécois. Nous nous sommes donc tournées vers les programmes ministériels et les programmes d'études universitaires en enseignement du français au secondaire afin de brosser un portrait des pratiques possibles d'enseignement du théâtre dans les classes de français au Québec. Cette analyse de documents officiels québécois relatifs à l'enseignement du théâtre en classe nous a également permis de faire la lumière sur les écarts possibles entre les pratiques recommandées par les programmes ministériels et la formation reçue par les futurs maitres.

\section{L'enseignement du théâtre en classe de français langue de scolarité au Québec}

Il existe à notre connaissance peu d'études (sinon aucune) portant sur la question de l'enseignement du théâtre et par le théâtre en classe de français langue d'enseignement au secondaire au Québec. Ainsi, on ne connait pas les pratiques des enseignants, ni l'éventuel impact de celles-ci sur les apprentissages linguistiques des élèves. Dans notre revue des écrits, nous avons bien recensé une étude de type exploratoire menée au secondaire (St-Cœur, 2004), mais celle-ci a été réalisée au Nouveau-Brunswick, une province canadienne limitrophe du Québec majoritairement anglophone où le français est une langue minoritaire. Cette recherche a permis de mieux connaitre les perceptions des enseignants de français au secondaire en milieu francophone minoritaire, au sujet de l'intégration des activités d'art dramatique dans leur enseignement. Les entretiens semi-dirigés menés auprès des dix enseignantes participant à l'étude montrent que peu d'élèves sont motivés pour apprendre le français dans leur milieu. Selon les enseignantes, les activités dites authentiques seraient le principal type d'activité pouvant influencer positivement la motivation des élèves, l'activité de type théâtral arrivant au deuxième rang. Ainsi, une majorité de répondantes a confirmé avoir 
perçu des effets positifs à la suite d'activités théâtrales, notamment une augmentation globale de la motivation et de la confiance en soi des élèves, ce qu'ont également montré les études rapportées dans la section précédente. Plusieurs enseignantes ont cependant affirmé se sentir démunies lorsque venait le temps d'utiliser des stratégies théâtrales. Cet aspect constitue précisément une des difficultés qui expliqueraient la sous-exploitation du théâtre en classe. Une étude comme celle de St-Cœur (2004) demande à être menée en contexte québécois afin de mieux connaitre non seulement les pratiques d'enseignement du théâtre, mais aussi le rapport au thêâtre et à son enseignement chez les enseignants du secondaire.

Pour l'instant, en l'absence d'études spécifiques sur la question, nous tenterons d'établir un portrait de la situation en analysant, d'une part, le contenu des programmes de formation ministériels et, d'autre part, la composition des programmes universitaires de formation des maitres en français langue première au secondaire.

\subsection{Le programme ministériel}

Les programmes de formation ministériels présentent les contenus et les démarches à mettre en œuvre dans l'enseignement/apprentissage des disciplines et servent de guide aux enseignants et futurs enseignants quant aux compétences à développer chez leurs élèves. Par exemple, pour le domaine des arts et celui des langues, le Programme de formation de l'école québécoise (désormais PFEQ) identifie des visées communes : structuration de l'identité ; construction d'une vision du monde; développement du pouvoir d'action (MELS, 2006).

Nous avons donc analysé le PFEQ des premier et deuxième cycles du secondaire, afin de tenter de décrire les pratiques que préconise ce programme pour ce qui est de l'enseignement du théâtre en classe de français au secondaire. Cette analyse a été réalisée en deux étapes. La première a consisté à comptabiliser le nombre d'occurrences du mot «théâtre» dans le document ministériel. Nous avons ainsi répertorié 8 emplois du terme dans le programme du premier cycle, contre 37 apparitions dans le programme du deuxième cycle.

La deuxième étape a consisté à reporter chacune de ces occurrences dans différentes catégories d'analyse. Cette classification a été réalisée en analysant le contexte d'apparition du mot «théâtre», dans tout le contenu des programmes, ce qui nous a permis de dégager sept catégories de situations. Le tableau suivant illustre le nombre d'occurrences par catégorie, pour chacun des deux cycles. 


\begin{tabular}{|c|c|c|c|}
\hline & Catégorie & $\begin{array}{l}\text { Nombre } \\
\text { d'occurrences } \\
\text { au } 1^{\text {er }} \text { cycle }\end{array}$ & $\begin{array}{l}\text { Nombre } \\
\text { d'occurrences } \\
\text { au } 2^{\mathrm{e}} \text { cycle }\end{array}$ \\
\hline 1 & Le théâtre comme sortie à caractère culturel. & 1 & 1 \\
\hline 2 & Le théâtre comme possible sphère d'emploi. & 1 & 0 \\
\hline 3 & $\begin{array}{l}\text { Le genre théâtral envisagé comme une façon } \\
\text { de diversifier les œuvres lues. }\end{array}$ & 3 & 4 \\
\hline 4 & $\begin{array}{l}\text { L'écoute d'une pièce de théâtre comme façon } \\
\text { d'améliorer les compétences langagières de } \\
\text { l'élève (en lien direct avec une ou plusieurs } \\
\text { des trois compétences : lire, écrire, communi- } \\
\text { quer oralement). }\end{array}$ & 3 & 0 \\
\hline 5 & $\begin{array}{l}\text { La lecture et/ou écriture d'une pièce de } \\
\text { théâtre comme façon d'améliorer les compé- } \\
\text { tences langagières de l'élève (en lien direct } \\
\text { avec une ou plusieurs des trois compétences). }\end{array}$ & 0 & 23 \\
\hline 6 & $\begin{array}{l}\text { Le théâtre comme activité parascolaire } \\
\text { contribuant à l'amélioration des compétences } \\
\text { langagières. }\end{array}$ & 0 & 1 \\
\hline 7 & $\begin{array}{l}\text { Le mot «théâtre» est utilisé pour expliquer } \\
\text { ou illustrer un propos. }\end{array}$ & 0 & 6 \\
\hline
\end{tabular}

Tableau 1. - Catégorisation des occurrences du mot «théâtre» dans le programme de formation ministériel du Québec (PFEQ).

Voici quelques exemples d'énoncés du programme qui ont été classés dans chacune des sept catégories :

- Catégorie $1:$ «[...] la fréquentation guidée de lieux de culture (théâtre, musée, galerie, bibliothèque, exposition scientifique ou artisanale, foire du livre, maison de la culture, salle de presse, station de télévision communautaire, etc.) amène l'élève à se donner les clés nécessaires pour accéder à des œuvres diversifiées et découvrir les multiples facettes de l'univers culturel de sa région.» (Ibid., p. 8)

- Catégorie 2: «L'élève découvre peu à peu que de nombreux métiers et professions présentent des attentes élevées à l'égard des compétences langagières. C'est notamment le cas dans les milieux de l'édition, de la communication, de la science, de la technologie, des relations publiques, de l'enseignement, du théâtre et de la chanson.» (Ibid., p. 15) 
- Catégorie 3: «Même si la lecture accompagnée peut s'avérer nécessaire pour des œuvres plus exigeantes, il n'est pas souhaitable que tous les élèves lisent uniquement les mêmes livres. Le fait de leur proposer une grande variété d'œuvres favorise la diversification et l'enrichissement des repères qu'ils se construisent, que ce soit des genres de textes, des œuvres d'hier et d'aujourd'hui, des auteurs du Québec, de la France ou de la francophonie, des univers particuliers, des procédés d'écriture et même des transpositions d'œuvres au théâtre, au cinéma, etc.» (Ibid., p. 20)

- Catégorie 4: «Inscrite à l'intérieur de véritables situations de communication, l'écoute conduit l'élève à faire des apprentissages sur le monde, sur lui-même et sur la langue orale. Ainsi, l'enseignant peut lui proposer un rôle de recherchiste, d'observateur ou de critique. Il diversifie les conditions et les objets d'écoute. Celle-ci peut, par exemple, s'effectuer en direct ou en différé, impliquer ou non le recours aux technologies numériques, être centrée sur le traitement d'un sujet dans un documentaire ou encore sur les caractéristiques des personnages d'un film ou d'une pièce de théâtre.» (Ibid., p. 120)

- Catégorie 5: «Reconnaitre et utiliser des marques qui relient le texte à un genre oral (discussion, entrevue, conférence, documentaire, message publicitaire; monologue, chanson; etc.) ou écrit (chronique, lettre ouverte, éditorial, poème, pièce de théâtre, nouvelle littéraire, bande dessinée; etc.).» (Ibid., p. 124) ${ }^{1}$

- Catégorie 6 : «Le conseil d'élèves a confié à une classe de français la préparation d'une campagne publicitaire entourant la présentation, à l'école, d'une pièce de théâtre et la dégustation de produits régionaux au cours de l'entracte. Les élèves coopèrent pour planifier le travail, pour trouver des idées originales, pour choisir des procédés linguistiques qui contribuent à capter l'attention des lecteurs et pour se donner des règles afin d'assurer la contribution harmonieuse de chacun à la réalisation des tâches.» (Ibid., p. 26)

1. Tous les énoncés du programme touchent la lecture, mais certains touchent également l'écriture. 
- Catégorie 7: «Le sens attribué à l'expression "œuvre littéraire" peut varier. Il peut renvoyer à un type d'ouvrage (ex. album, recueil), à un texte en particulier (ex. roman, poème, pièce de théâtre) ou encore à l'ensemble de la production d'un écrivain.» (Ibid., p. 149)

Ces informations ne nous donnent évidemment pas d'indices sur les pratiques réelles d'utilisation du théâtre en classe de français. Cependant, nous pouvons mettre en relief les pratiques que recommande le ministère de l'Éducation du Loisir et du Sport (MELS). Celles-ci pourraient être résumées ainsi : au premier cycle, il s'agirait plutôt d'un aperçu général du théâtre qui toucherait davantage le développement global de l'adolescent. Les apprentissages ne viseraient donc pas seulement l'amélioration directe des compétences langagières, mais également le développement culturel et professionnel (le théâtre est présenté comme une sphère possible d'emploi). Cette initiation semble servir à préparer les élèves au deuxième cycle, où le théâtre occupe une place plus importante, notamment en matière d'amélioration des compétences langagières. Une utilisation du théâtre telle que prescrite au deuxième cycle s'inscrit précisément dans la perspective que nous défendons, soit un enseignement du français par le théâtre.

En plus du PFEQ, nous avons analysé un autre document ministériel, qui sert de guide aux enseignants pour la progression des contenus des disciplines dispensées dans le secondaire au Québec, soit la Progression des apprentissages au secondaire, section «Domaine des langues - français, langue d'enseignement» (MELS, 2011). Nous nous sommes plus particulièrement penchées sur la section intitulée «Le texte de théâtre (sous la rubrique «Les modes de discours»). Les renseignements qui ressortent de ce document concordent avec l'analyse précédente : même si la découverte du texte de théâtre est encouragée au primaire (élèves de 6 à 12 ans) et au début du secondaire (élèves de 12 à 17 ans), les apprentissages relatifs au texte de théâtre ne sont réellement prescrits qu'en quatrième et cinquième secondaires (élèves âgés de 15 à 17 ans). Plus précisément, les principales compétences concernées par le théâtre comme mode de discours sont réparties sous trois grands thèmes : (1) situation de communication; (2) organisation du texte dramatique; (3) cohérence et organisation du texte. Sous ces trois thèmes s'inscrivent donc des compétences telles que : (1) se situer en tant que destinataire (lecteur ou spectateur d'une pièce de théâtre), tenir compte du contexte de production, etc.; (2) distinguer le dialogue 
des didascalies, distinguer les types de prises de parole : monologue (aparté, soliloque, tirade), réplique, dégager les caractéristiques de genres et des critères d'appréciation en comparant l'univers construit avec d'autres œuvres, etc.; (3) reconnaitre les marques d'organisation du texte : le découpage en actes, scènes et tableaux, les changement de temps, de lieux et de décor, l'arrivée ou le départ d'un personnage, etc.

Enfin, rappelons encore une fois qu'il s'agit ici de contenus et de pratiques prescrites par le ministère et non de pratiques d'enseignement effectives, qui, à notre connaissance, ne sont pas encore documentées. Il n'en reste pas moins que ces textes ministériels sont susceptibles de guider les pratiques des enseignants. Un autre facteur pouvant exercer une influence sur l'enseignement du français par le théâtre en classe est la formation reçue par les futurs maitres. Dans le cadre de cet article, nous nous intéressons plus précisément à la formation initiale en contexte universitaire québécois.

\subsection{La formation initiale des maitres}

Comme nous l'avons mentionné précédemment, nous en savons peu sur les pratiques d'enseignement du théâtre et par le théâtre en classe de français au Québec. Néanmoins, un regard vers la formation des maitres peut nous éclairer quant aux types de pratiques d'enseignement proposées. Par exemple, en France, Bernanoce (2013) a précisément abordé la question de la formation initiale des maitres en lien avec le théâtre en classe de français. Elle souligne que la majorité des enseignants de français ne seraient pas formés pour enseigner le théâtre. Quelques conséquences peuvent en découler : (1) l'enseignement est peu uniforme, tant dans la fréquence des activités théâtrales que dans leur qualité; (2) il est difficile d'évaluer les retombées de cette formation; (3) les enseignants ressentent de l'insécurité parce qu'ils se sentent peu outillés. Nous faisons l'hypothèse que la formation universitaire des futurs enseignants au Québec entraine des conséquences semblables.

Cette section de l'article servira principalement à dresser un portrait global de la formation offerte aux futurs maitres par les différentes universités québécoises en lien avec l'enseignement du français au secondaire. Plus précisément, nous centrerons notre analyse sur les liens qui y sont tissés avec le théâtre. Nous tenterons d'évaluer si les étudiants reçoivent un enseignement uniforme d'une université à l'autre, et s'ils sont suffisamment outillés pour répondre aux exigences ministérielles telles que décrites à la section précédente. Afin de dresser ce tableau, 
nous avons analysé les programmes universitaires en enseignement du français $^{2}$ langue d'enseignement au secondaire des neuf universités québécoises (UQAM, UdeM, UQTR, UQAR, UQAT, UQO, UQAC et USherbrooke) en étudiant leurs descriptifs de cours (disponibles en ligne). Cette analyse a pour but de vérifier si le contenu des cours dispensés aux futurs enseignants dans ces programmes universitaires est conforme au contenu du programme ministériel concernant les apprentissages des élèves du secondaire.

Nos critères d'analyse sont les suivants (tableau 2) : (1) nombre de cours en lien direct avec le théâtre et le texte de théâtre (texte et/ou activité théâtrale); (2) nombre de cours en lien avec la didactique du théâtre (texte et/ou activité théâtrale); (3) contenu de ces cours, le cas échéant.

Comme il ne s'agit que d'une étude de surface réalisée grâce aux descriptifs de cours affichés sur le site des universités, l'analyse demeure assez sommaire. Elle nous permet cependant de comparer les programmes des universités en lien avec l'enseignement du théâtre et de la didactique du théâtre.

De cette observation, il ressort que : (1) quatre universités sur neuf offrent un cours portant entièrement sur le théâtre; (2) deux universités offrent des cours optionnels consacrés au théâtre (UdeM et ULaval); (3) à l'intérieur des quatre programmes universitaires comportant un cours obligatoire sur le théâtre, deux (ceux de l'UQTR et de l'UQAR) enseignent à la fois le texte, les formes et les fonctions du spectacle et deux (ceux de l'UQAM et de l'ULaval) n'abordent que la question du texte; (4) aucun cours de ces neuf universités n'aborde la didactique du théâtre ou n'envisage explicitement des perspectives de réinvestissement en classe.

Le constat que nous pouvons en tirer est qu'à la suite de leurs études universitaires, les enseignants de français au secondaire auront reçu un enseignement non uniforme : certains auront eu la chance d'explorer le texte théâtral et l'activité théâtrale ${ }^{3}$, alors que d'autres auront, au mieux, abordé le texte dramatique.

2. Au niveau du baccalauréat.

3. Le terme «activité théâtrale» est utilisé ici en référence à toute activité ou atelier dramatique sans inclure nécessairement la finalité du spectacle. Par exemple, il peut s'agir d'un exercice pratique sur la projection de la voix qui aurait comme objectif de peaufiner la compétence orale. 


\begin{tabular}{|c|c|c|c|}
\hline & $\begin{array}{c}\text { Nombre de cours en } \\
\text { lien direct avec le } \\
\text { théâtre }\end{array}$ & Contenu & $\begin{array}{l}\text { Nombre de } \\
\text { cours en lien } \\
\text { direct avec la } \\
\text { didactisation } \\
\text { du théâtre }\end{array}$ \\
\hline UQAM & $\begin{array}{l}1: \text { Histoire et } \\
\text { esthétique du } \\
\text { texte dramatique } \\
\text { (LIT6245) }\end{array}$ & $\begin{array}{l}\text { Aperçu historique, forme, } \\
\text { esthétique, étude des genres } \\
\text { et composantes propres au } \\
\text { texte théâtral }\end{array}$ & 0 \\
\hline UdeM & 0 & - & 0 \\
\hline UQTR & $\begin{array}{l}1: \text { Le langage } \\
\text { dramatique } \\
\text { (ARD1008) }\end{array}$ & $\begin{array}{l}\text { Aperçu historique, structure, } \\
\text { modes et esthétiques du texte + } \\
\text { Mise en scène, et ensembles } \\
\text { signifiants du spectacle }\end{array}$ & 0 \\
\hline UQAR & $\begin{array}{l}1: \text { Approches du } \\
\text { théâtre (MET20210) }\end{array}$ & $\begin{array}{l}\text { Le texte : répliques et } \\
\text { didascalies }+ \\
\text { Genre, formes, langage drama- } \\
\text { tique, systèmes sémiotiques du } \\
\text { spectacle }\end{array}$ & 0 \\
\hline UQAC & 0 & - & 0 \\
\hline UQAT & 0 & - & 0 \\
\hline UQO & 0 & - & 0 \\
\hline USherb. & 0 & - & 0 \\
\hline ULaval & $\begin{array}{l}1: \text { Genres littéraires } \\
\text { (roman et théâtre) }\end{array}$ & $\begin{array}{l}\text { Dramaturgie et analyse de } \\
\text { texte, perspective littéraire }\end{array}$ & 0 \\
\hline
\end{tabular}

Tableau 2. - Comparaison des programmes universitaires québécois de baccalauréat en enseignement du français langue d'enseignement au secondaire en lien avec le théâtre et sa didactique.

L'ajout d'un cours d'introduction au théâtre dans l'ensemble des programmes universitaires permettrait, selon nous, de répondre aux lacunes soulevées par notre analyse. Un tel cours contribuerait par exemple à : (1) uniformiser la formation des enseignements de français en lien avec le théâtre à travers toute la province de Québec; (2) offrir des outils didactiques aux futurs enseignants en lien avec le théâtre en classe, leur permettant d'être plus à l'aise avec cette discipline et son enseignement (enseignement du théâtre et enseignement par le théâtre).

Comme l'un de nos principaux constats est que la formation initiale propose peu de pistes didactiques d'utilisation du théâtre en classe de 
français, nous suggérons, dans la section suivante, quelques propositions didactiques destinées aux enseignants de français au secondaire et pouvant être adaptées pour l'enseignement du français langue seconde ou étrangère.

\section{Pistes didactiques pour exploiter le théâtre en classe de français}

Les exercices que nous proposons dans cette section ont tous été testés maintes fois en classe et ont été choisis afin d'échapper à de réelles contraintes techniques que nous allons d'abord présenter brièvement.

\subsection{Diverses contraintes techniques}

En nous appuyant sur notre expérience personnelle d'enseignement et sur des témoignages d'enseignants recueillis informellement lors de l'animation d'une séance de formation continue (Dubois \& Tremblay, 2014), nous avons identifié trois types de contraintes techniques pouvant, selon nous, expliquer la sous-exploitation de l'activité théâtrale en classe de français : les contraintes financières, la gestion de l'espace, la gestion comportementale.

Premièrement, une des contraintes à ne pas passer sous silence est évidemment la possibilité de restrictions financières. Par exemple, l'achat de textes, qu'il s'agisse de livres ou de droits de reproduction, peut représenter des couts importants. Il est fort possible que l'achat d'autres genres, tel que le roman, soit privilégié lorsque le budget est limité. En effet, le roman est un genre qui est généralement préféré aux autres (Dardaillon, 2009).

Deuxièmement, il peut être déstabilisant pour un enseignant de devoir changer la disposition spatiale régulière de la classe pour la mise en place d'activités théâtrales : déplacer du mobilier avant et après l'activité peut entrainer une perte de temps, en plus de créer une éventuelle désorganisation au sein du groupe. En outre, la gestion de classe n'est pas la même lorsque les élèves sont debout et en action dans l'espace plutôt que sagement assis derrière leur bureau. Un espace de travail différent est susceptible de déstabiliser les élèves (et l'enseignant) qui n'ont pas forcément l'habitude de se réunir dans un lieu ainsi configuré. Ainsi, si la nouveauté contribue à créer une ambiance euphorique, elle peut aussi, à l'inverse, gêner certains élèves. Lorsque nous incitons nos élèves à sortir de leur «zone de confort», il faut anticiper autant de 
réactions qu'il y a d'élèves. Il y a fort à parier que chaque classe compte quelques élèves qui risquent d'être embarrassés à l'idée de devoir «faire du théâtre». Comment se comporter avec ces élèves que nous voulons aider à s'extérioriser? Certes, il faut les encourager à participer sans les forcer, mais il est surtout important de les guider progressivement vers une prise de parole ludique. Quant aux plus agités, nous devons les guider vers le respect qu'exige toute activité de classe en groupe : écoute des camarades, prise de parole au moment opportun, etc.

Ces considérations nous amènent à proposer un certain nombre de pistes didactiques qui ont été créées afin de pouvoir exploiter le théâtre en classe de français, sans exiger de moyens financiers particuliers. Les activités peuvent aussi être réalisées sans trop transformer l'espace de la classe. Enfin, elles devraient convenir à tous les types d'élèves, du plus timide au plus agité.

Nous présenterons les activités en en proposant d'abord une synthèse. Nous détaillerons ensuite les objectifs, la (les) compétence(s) visée(s), la durée approximative de la réalisation, le matériel à préparer (s'il y a lieu) et nous terminerons en proposant quelques variantes possibles de l'activité.

Il est à noter que les propositions présentées visent l'amélioration des compétences ministérielles présentées dans les programmes (compétence à l'oral, en écriture et en lecture) tout en cherchant à contrer un certain flou didactique laissé par la formation initiale.

\subsection{L'exercice du sous-texte}

La notion de sous-texte, dans le monde théâtral, renvoie aux implicites du texte dramatique, à ce que le texte ne dit pas, mais que l'on comprend grâce à l'interprétation d'un comédien (Pavis, 2002). L'exercice du sous-texte fait appel à la maitrise de compétences verbales et paraverbales et permet d'amener les élèves à les développer à travers une courte prise de parole.

- OBJECTIFS : l'exercice du sous-texte a pour objectif d'introduire ou d'approfondir des notions telles que le volume, le débit, le regard, la gestuelle, etc. Il sert également à concrétiser ces objets d'enseignement et à faire comprendre aux élèves la pertinence de les maitriser.

- COMPÉTENCE VISÉE : expression orale.

- DURÉE DE RÉALISATION : environ 15 minutes. 
- MATÉRIEL : bouts de papier sur lesquels on trouve le nom d'une émotion (colère, surprise, honte, amour, joie, tristesse, etc.).

\section{- ÉTAPES DE RÉALISATION :}

1. Organiser la classe en cercle ou de sorte que tous les élèves puissent se voir.

2. Faire tirer à chaque élève un papier sur lequel apparait le nom d'une d'émotion. L'élève ne révèle pas l'émotion qui lui est attribuée.

3. Choisir une courte phrase. Cette phrase sera dite par tous les élèves à tour de rôle. Le choix se fait indifféremment par l'enseignant ou par le groupe. Il est suggéré de choisir quelque chose de simple et qui commencerait avec le pronom «je», tel que : «Je suis fatigué(e)», «J'adore les devoirs», etc.

4. À tour de rôle, chaque élève doit énoncer au reste du groupe la phrase choisie (la même pour tous) selon l'émotion tirée (différente pour chaque élève) en adaptant le volume de sa voix, son débit, son regard, sa gestuelle, etc., afin que les autres élèves puissent identifier l'émotion avec laquelle la phrase est dite.

5. Les autres, à main levée, tentent de deviner l'émotion exprimée par l'élève en justifiant leur réponse. Par exemple : «Je crois que l'émotion exprimée par Untel est la colère parce que lorsqu'il a prononcé sa phrase, il a utilisé un regard menaçant (ses sourcils étaient froncés), son débit était plutôt saccadé et le volume de sa voix était assez élevé. De plus, Untel avait les bras croisés, ce qui nous donnait un indice supplémentaire au sujet du sentiment exprimé.»

6. L'enseignant complète soit en introduisant les notions relatives aux objets d'enseignement soit en faisant des rappels de ce qui a été vu en classe.

\section{- PARMI LES VARIANTES POSSIBLES :}

1. La configuration de la classe peut demeurer inchangée par rapport à la configuration habituelle. Les élèves peuvent se déplacer à l'avant de la classe.

2. Les élèves choisissent leur propre émotion. Il peut s'agir également d' «intentions». Par exemple, «avoir l'intention d'avouer quelque chose». 
3. La phrase est tirée de l'ouvrage qui est présentement à l'étude, peu importe le genre littéraire.

Cet exercice relève véritablement d'un enseignement par le théâtre, car c'est grâce à une activité d'ordre dramatique que l'on vise l'amélioration des compétences langagières. Des notions de théâtre peuvent bien sûr être abordées. Par exemple, il serait intéressant d'amener l'élève à prendre conscience du rapport entre l' «acteur» et le «spectateur» lorsqu'il interprète la courte phrase en faisant complètement face aux autres élèves plutôt que de biais ou de dos. En effet, nous rencontrons régulièrement des élèves qui, d'emblée, ne s'adressent pas à leur public directement.

\subsection{La ritualisation du Théâtre des lecteurs}

L'activité du Théâtre des lecteurs consiste en une lecture à voix haute et en groupe d'une pièce (ou d'un extrait). Les «rôles » sont assignés aux élèves. Ses bienfaits, relevés précédemment, ont été mis en lumière par plusieurs recherches (Smith, 2011; Mendes, 2012 ; McFadden, 2010 ; La Von Bridges, 2006). Cet exercice permet une introduction simple à la pratique théâtrale sans déranger l'organisation spatiale de la classe et en tenant compte de la sensibilité des élèves.

- OBJECTIFS : 1'exercice du Théâtre des lecteurs a pour objectifs : (1) d'améliorer la fluidité des élèves en lecture; (2) de faciliter la compréhension en lecture des élèves grâce à une coconstruction de sens; (3) de favoriser l'acquisition de différentes habiletés à l'expression orale telles que l'articulation et la projection, le débit, etc. (l'enseignant choisira les objets d'enseignement sur lesquels il souhaite faire porter l'attention puisqu'un grand nombre sont sollicités par l'exercice); (4) de permettre aux élèves de se dégager de leur possible gêne face à la prise de parole en classe : la ritualisation de l'activité et un entrainement régulier peuvent en effet contribuer à les mettre à l'aise.

- COMPÉTENCES VISÉES : lecture et expression orale.

- DURÉE DE RÉALISATION : environ 15 minutes (au moins une fois par semaine).

\section{- ÉTAPES DE RÉALISATION :}

1. Choisir une œuvre théâtrale à lire. Par exemple : Premières de classe (Casey Kurti, traduit par Tremblay; 4 personnages 
principaux), Sortie de secours (collectif; 23 personnages), Dans les dunes de Tadoussac (Jean-Pierre Ronfard; 20 personnages), etc. ${ }^{4}$.

2. Définir la fréquence et la durée des lectures.

3. Attribuer des rôles à chaque élève avant de commencer la lecture en considérant les caractéristiques de chacun (timidité, sensibilité, etc.). Par exemple, les plus timides peuvent commencer avec un plus petit rôle et, en cours de processus, s'en voir attribuer un plus important.

4. Procéder à la lecture du texte en groupe en demandant à chaque élève de lire les répliques du personnage qui lui a été attribué.

5. Donner des commentaires constructifs pendant l'activité selon les objets d'enseignement travaillés (par exemple : débit, volume, articulation, etc.).

6. Assurer un retour simple à la fin de chaque séance sous forme d'échanges rétroactifs en lien avec les objectifs de départ.

\section{- PARMI LES VARIANTES POSSIBLES :}

1. Choisir des extraits d'œuvres avant de se lancer dans la lecture d'une œuvre complète, ce qui permettra de se familiariser avec l'activité d'abord.

2. S'il y a trop d'élèves pour le nombre de personnages, il est possible de procéder à deux ou trois distributions des mêmes rôles et alterner (soit d'une séance à une autre soit au cours d'une même séance).

Cet exercice peut également servir à l'enseignement du théâtre et à l'enseignement par le théâtre. Dans le premier cas, il s'agit de l'occasion idéale d'aborder le texte dramatique et ses particularités (didascalies, dialogue, découpage des scènes, etc.). Dans le deuxième cas, il s'agit de l'occasion idéale de se focaliser sur les objets d'enseignement du français : compétences en lecture (dont la fluidité et la compréhension) et compétences à l'oral (projection de la voix, articulation, diction, posture, etc.).

4. Nous suggérons de consulter le site internet du Centre des auteurs dramatiques (CEAD : <www.cead.qc.ca>) où il est possible d'effectuer une recherche de textes qui tient compte du nombre de personnages. 


\subsection{La compréhension grâce à un texte blanc}

Le texte blanc est un texte de théâtre dans lequel les répliques ne sont pas attribuées à des personnages (soit par une intention volontaire de l'auteur, soit parce que les noms ont été retirés à des fins didactiques). Par exemple, dans cet extrait d'Arlequin, Serviteur de deux maitres (Acte 1, tableau I) ${ }^{5}$ les noms des personnages et les didascalies ont été effacés :

- Je crois que ce garçon est un niais.

- Il m'a plutôt l'air d'un farceur.

- Votre Seigneurie est-elle mariée?

- Hélas, non, monsieur.

- Veuillez me dire qui vous êtes, sinon allez-vous-en!

- Si c'est tout ce que vous désirez savoir, je vais vous satisfaire en deux mots. Je suis le valet de mon maitre. Donc, pour revenir à nos moutons...

- Mais qui est votre maitre?

- C'est un étranger qui voudrait venir vous rendre une petite visite.

- Nous causerons de cette histoire de mariage.

Voici les indications pédagogiques concernant cette activité visant la compréhension à travers le texte blanc.

- OBJECTIF : l'exercice du texte blanc, un texte dont on a retiré le nom des personnages, est un moyen ludique d'aborder la question de la compréhension et de l'interprétation avec des élèves. Ceux-ci devront éventuellement attribuer les répliques à des personnages. Pour y arriver, ils doivent comprendre certains éléments du texte blanc afin qu'une fois les noms des personnages ajoutés, le texte demeure logique. Ils doivent également interpréter le sens de chaque réplique avant d'attribuer les noms des personnages. Ainsi, plusieurs résultats sont possibles.

- COMPÉTENCE VISÉE : lecture.

- DURÉE DE RÉALISATION : environ 30 minutes.

- ÉTAPES DE RÉALISATION :

1. Former de petites équipes (2 à 4 élèves).

2. Attribuer le même texte blanc à toutes les équipes.

5. Carlo Goldoni (1745), traduit de l'italien par Valeria Tasca (1998). 
3. Expliquer ce qu'est un texte blanc.

4. Demander aux élèves de lire le texte et d'attribuer des noms (personnages) à chaque réplique.

5. Demander à un responsable par équipe de présenter leur travail au reste de la classe et comparer les résultats entre eux (cohérence textuelle, relations entre les personnages, crédibilité de la situation, etc.).

6. Aborder les concepts de compréhension et d'interprétation grâce à une discussion métacognitive en s'appuyant sur l'expérience vécue (par exemple les notions de cohérences, d'inférences, etc.).

\section{- PARMI LES VARIANTES POSSIBLES :}

1. On peut imposer un nombre maximum de personnages pour faciliter la tâche, ou encore déterminer d'avance les personnages pour faciliter encore plus le travail.

2. Si les didascalies offrent peu d'indices, on peut imposer des contraintes (par exemple lieux, époques) afin de faciliter l'exercice.

Encore une fois, cette activité peut servir à l'enseignement du théâtre et à l'enseignement par le théâtre. Dans le premier cas, l'enseignant pourrait aborder, par exemple, la structure du texte dramatique. Dans le deuxième cas, l'enseignant peut, comme le titre l'indique, travailler la compréhension du texte, de même que d'autres compétences liées à la restitution orale.

\subsection{La compréhension grâce à la dramatisation}

Le processus de dramatisation porte sur la mise en dialogue, la création d'une tension dramatique et/ou la mise en relief de la dynamique de l'action, et ce, relativement au texte théâtral (Pavis, 2002).

- OBJECTIF : la dramatisation permet de vérifier la compréhension de l'élève sans nécessairement l'évaluer. Selon De Peretti (2013), ce type d'activité serait susceptible de modifier le rapport à la lecture et à l'écriture, particulièrement chez les élèves en difficulté. En effet, l'adaptation d'un texte exige que l'élève comprenne le texte initial, qu'il soit en mesure d'identifier les éléments prédominants du récit (personnages, lieux, actions, etc.) et qu'il sache faire des liens entre ces éléments. En quelque 
sorte, le texte dramatisé est tributaire du processus métacognitif de l'élève.

- COMPÉTENCES VISÉES : lecture et écriture.

- DURÉE DE RÉALISATION : environ 20 minutes.

- ÉTAPES DE RÉALISATION :

1. Former de petites équipes (2 à 4 élèves).

2. Présenter un court texte au choix (selon le niveau et le temps).

3. Demander aux élèves de faire une première lecture du texte et d'en relever les principaux éléments : action principale, personnages, lieu, etc.

4. Expliquer ce qu'est la dramatisation en modélisant à partir du texte, c'est-à-dire en faisant soi-même la dramatisation. Cela permettra aux élèves de mieux comprendre ce dont il s'agit.

5. Demander aux élèves de poursuivre l'exercice.

\section{- PARMI LES VARIANTES POSSIBLES :}

1. Demander à chaque équipe de procéder à la dramatisation d'une scène différente d'un même texte, de sorte qu'après une mise en commun, on arrive à l'histoire complète.

2. À la suite de cette activité, procéder à une séance de Théâtre des lecteurs pour présenter le résultat au reste de la classe.

Dans ce cas-ci encore, l'exercice peut servir à l'enseignement du théâtre et à l'enseignement par le théâtre. Dans le cas de l'enseignement du théâtre, l'enseignant peut choisir de travailler autant l'aspect textuel que les conventions scéniques. Dans le cas de l'enseignement par le théâtre, il peut choisir de travailler des objets d'enseignement propres aux compétences scripturales et de lecture.

\section{Conclusion}

Dans cette contribution, nous avons tenté de dresser un état des lieux de l'enseignement par le théâtre en français au secondaire au Québec, à partir d'une analyse des programmes ministériels et des programmes universitaires de formation des maitres. D'autres études (entretiens, pratiques de classes filmées, etc.) sont nécessaires afin de connaitre les pratiques réelles d'enseignement du français par le théâtre au Québec. Malgré tout, notre analyse a permis de montrer que la formation initiale des maitres ne serait pas tout à fait au diapason des propositions 
formulées dans les programmes d'éducation ministériels : moins de la moitié des programmes offrent en effet aux futurs maitres de français un cours portant sur le théâtre (ne tenant pas compte d'autres cours, de littérature par exemple, où la question pourrait être abordée). Aucun de ces programmes, selon les descriptifs de cours consultés, n'évoque la question de la didactisation du théâtre, éludant de ce fait les perspectives d'investissement du théâtre en classe. Nous croyons que cela pourrait contribuer à expliquer certaines réticences des enseignants de français à utiliser le théâtre comme moyen d'enseignement, en plus des contraintes techniques précédemment mentionnées.

Les pistes proposées dans l'article visent à «répondre» à la possible méfiance des enseignants à l'égard d'une approche non conventionnelle qu'est l'approche par l'art et aux difficultés plus techniques que de telles pratiques peuvent poser. Nous espérons que nos propositions pourront ainsi contribuer à un changement de perspective, afin que l'enseignement du français dynamisé par le recours aux pratiques théâtrales se fasse au bénéfice d'un public de plus en plus vaste.

\section{RÉFÉRENCES BIBLIOGRAPHIQUES}

AdEn, Joëlle. (2009). La créativité artistique à l'école : refonder l'acter d'apprendre. Synergies Europe, 4, 173-180.

Audet, René \& Mercier, Andrée. (2004). La narrativité contemporaine au Québec : la littérature et ses enjeux narratifs. Québec, Canada : Les Presses de l'Université Laval.

Bernanoce, Marie. (2013). Le répertoire théâtral dans son contexte scolaire, à l'épreuve des genres et des esthétiques. Le français aujourd'hui, $180,27-38$.

BOMBARDIER, Louise et coll. (1984). Sortie de secours. Montréal, Canada : VLB éditeur.

CEAD. Site du Centre des auteurs dramatiques. Disponible en ligne sur <www.cead.qc.ca> (consulté le 8 mai 2015).

CHARLOt, Bernard. (1997). Du rapport au savoir. Éléments d'une théorie. Paris : Anthropos.

Dardaillon, Sylvie. (2009). Les albums de Béatrice Poncelet à la croisée des genres : expériences de lecture, enjeux littéraires et éducatifs, implications didactiques (Thèse de doctorat). Université François Rabelais, Tours.

De Peretti, Isabelle. (2013). Patrimoine littéraire et théâtre contemporain de jeunesse au cycle 3. Dans S. Ahr et N. Denizot (dir.), Les patrimoines 
littéraires à l'école, usages et enjeux (p. 51-57). Namur, Belgique : Presses universitaires de Namur.

Dubois, Joannie \& Tremblay, Ophélie. (2014). Coffre à outils pour enseigner le théâtre en classe de français. Communication orale présentée au congrès de l'Association québécoise des professeurs de français : «Le français, voie de communication », Sherbrooke, Canada.

Fonio, Filippo \& Genicot, Geneviève. (2011). The Compatibility of Drama Language Teaching and CEFR Objectives - Observations on a Rationale for an Artistic Approach to Foreign Language Teaching at an Academic Level. Scenario, 5(2), 75-89.

Goldoni, Carlo. (1745; 1998). Arlequin serviteur de deux maitres (V. Tasca, trad.). Paris : Flammarion.

Kamaruddin, Fadzliyati \& Kamarul Kabilan, Muhammad. (2010). Engaging Learner's Comprehension, Interest and Motivation to Learn Literature Using the Reader's Theatre. English Teaching: Practice and Critique, 9(3), 132-159.

Kurtti, Casey. (1993). Premières de classe (M. Tremblay, trad.). Montréal, Canada : Leméac.

Lachaise, Virginie. (2008). Les élèves à l'école du théâtre obligatoire. Jeu : revue de théâtre, 126(1), 105-107.

LA Von Bridges, Carolyn. (2008). Effects of Readers' Theatre on English Language Learners: A Strategy for Oral Language and Reading Improvement. TNTESOL Journal, 1, 21-30.

MacFadden, Pamela. (2010). Using Theatre Arts to Enhance Literacy Skills at the Second Grade Level (Thèse de doctorat). University of California, Los Angeles, États-Unis.

Magalhaes dos Reis, Maria da Glória. (2008). Le texte théâtral et le jeu dramatique dans l'enseignement du FLE. Synergies Espagne, 1, 153-162.

Mendes, Holly Marie. (2012). Improving Fluency in English Language Learners Through Readers' Theatre (Mémoire de maitrise). Saint Mary's College of California, Moraga, États-Unis.

MeIrIeU, Philippe (2002). Le théâtre et l'école : éléments pour une histoire, repères pour un avenir. Dans J.-C. Lallias (dir.), Le théâtre et l'école : histoire et perspectives d'une relation passionnée (p. 30-47). Arles : Actes Sud.

Ministère de L'Éducation, du Loisir et du Sport du Québec (MELS). (2006). Programme de formation de l'école québécoise. Québec, Canada.

Ministère de L'Éducation, du Loisir et du Sport du Québec (MELS). (2011). Progression des apprentissages au secondaire. Québec, Canada. Molière $(1673 ;$ 1965). Le Malade imaginaire. Dans Euvres complètes IV. Paris : Garnier Flammarion.

Pavis, Patrice (dir.). (2002). Le dictionnaire du théâtre. Paris : Armand Colin. 
Ronfard, Jean-Pierre. (1985). Dans les dunes de Tadoussac. Montréal, Canada : CEAD.

Simard, Denis. (2004). Éducation et herméneutique. Contribution à une pédagogie de la culture. Québec, Canada: Les Presses de l'Université Laval.

SimARD, Denis \& FALARDEAU, Érick. (2007). Le rapport à la culture des enseignants : proposition d'un cadre théorique. Nouveaux cahiers de la recherche en éducation, 10(2), 131-150.

SмIтн, Damon M. (2011). Readers' Theatre : Its Effectiveness in Improving Reading Fluency, Student Motivation and Attitude Toward Reading Among Second-Grade Students (Thèse de doctorat). Pennsylvania State University, State College, États-Unis.

SousA, David. (2004). Un cerveau pour apprendre : comment rendre le processus enseignement-apprentissage plus efficace. Montréal, Canada : Chenelière Éducation.

ST-Céur, Robert. (2004). Les perceptions des enseignants de français au secondaire en milieu francophone minoritaire au sujet de l'intégration des activités d'art dramatique dans leur enseignement et les perceptions de ces mêmes enseignants au sujet de la formation qu'ils ont reçue (Mémoire de maitrise). Université de Moncton, Moncton, NouveauBrunswick, Canada.

Tremblay, Michel. (1972). En pièces détachées. Montréal, Canada : Leméac. 